\title{
Rivaroxaban reduces length of hospital stay in patients with symptomatic venous thromboembolism
}

The phase III EINSTEIN deep vein thrombosis (DVT) and EINSTEIN pulmonary embolism (PE) trials demonstrated the potential of oral rivaroxaban (Xarelto, Bayer) - $15 \mathrm{mg}$ twice daily for 21 days, followed by $20 \mathrm{mg}$ once daily - for the treatment of venous thromboembolism (VTE), a term that embraces DVT and PE. A subsequent study by van Bellen et al.. ${ }^{[1]}$ published in Current Medical Research and Opinion in 2014, was undertaken to assess the length of initial hospitalisation in patients presenting with either symptomatic DVT or PE using hospitalisation records from these trials.

The authors found that overall 52\% of EINSTEIN DVT patients and 90\% of EINSTEIN PE patients were admitted to hospital. The proportion of hospitalised DVT patients with a length of stay 5 days or fewer, receiving rivaroxaban, was $54 \%$ compared with $31 \%$ for those receiving enoxaparin/vitamin K antagonist (VKA), the current standard of care for the treatment of patients with symptomatic DVT and PE. For patients with PE, the corresponding values were $45 \%$ and $33 \%$. Stays of 6 - 10 days were observed in $29 \%$ of rivaroxaban-treated patients compared with $45 \%$ for enoxaparin/VKA-treated patients for DVT. For patients with PE, these values were $39 \%$ and $46 \%$ in the rivaroxaban and enoxaparin/ VKA groups, respectively. Overall, length of stay was significantly shorter in the rivaroxaban group, compared with the enoxaparin/VKA group across all analyses performed $(p<0.0001)$.

VTE is associated with significant morbidity and mortality and therefore carries a considerable healthcare burden. Rivaroxaban is as effective as enoxaparin/VKA for the treatment of acute symptomatic DVT or PE, with the additional benefit of significantly reducing the period of hospitalisation in patients being treated for an initial DVT or PE. 'Coupled with improved patient treatment satisfaction and no requirement for routine monitoring or dose adjustment, this presents strong advantages for treating patients with VTE with rivaroxaban', the authors wrote. They concluded that a single-drug regimen with rivaroxaban may reduce the burden on healthcare systems and patients by providing effective and well-tolerated treatment. 'The convenience of a single-drug approach with oral rivaroxaban has the potential to allow discharge based on a patient's clinical condition and to facilitate the transition from in-hospital to outpatient care. [...] However, assessment of patient risk is still warranted to identify candidates who can safely receive outpatient treatment, and patient monitoring is essential to ensure adherence to the specified dosing regimen.'

\section{Reference}

1. van Bellen B, Bamber L, Correa de Carvalho F, et al. Reduction in the length of stay with rivaroxaban as a single-drug regimen for the treatment of deep vein thrombosis and pulmonary embolism. Curr Med Res Opin 2014; 30(5):829-837. [http://dx.doi.org/10.1185/03007995.2013.879439]

For full prescribing information, refer to the package insert approved by the Medicines Regulatory Authority (MCC).

\section{PHARMACOLOGICAL CLASSIFICATION: A.8.2 Anticoagulants.}

S4 XARELTO' 10. Reg. No.: 42/8.2/1046. Each film-coated tablet contains rivaroxaban $10 \mathrm{mg}$.

INDICATION: Prevention of VTE in patients undergoing major orthopaedic surgery of the lower limbs.

S4 XARELTO 15 and XARELTO' 20. Reg. No.: 46/8.2/0111 and 46/8.2/0112. Each film-coated tablet contains rivaroxaban $15 \mathrm{mg}$ or $20 \mathrm{mg}$, respectively. INDICATIONS: Prevention of stroke and systemic embolism in patients with non-valvular atrial fibrillation; Treatment of DVT and for the prevention of recurrent DVT and PE; Treatment of PE and for the prevention of recurrent PE and DVT.

Bayer (Pty) Ltd, Co. Reg. No.: 1968/011192/07, 27 Wrench Road, Isando, 1609. Tel: 0119215044 Fax: 0119215041.

L.ZA.GM.06.2014.1007
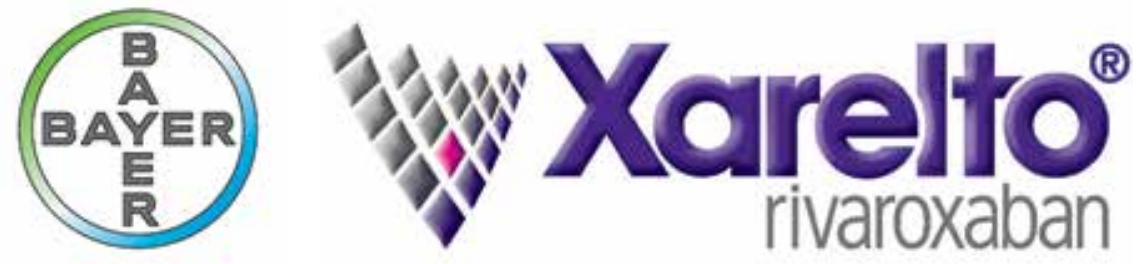

๑ Bayer HealthCare Pharmaceuticals June 2014 


\section{NEW Antistatic Chamber: small, solid and effective}

Aspen is proud to announce the launch of VORTEX ${ }^{\circledR}$, an innovative aluminium antistatic holding chamber with 'cyclone twist' principle, as an addition to our respiratory portfolio.

VORTEX ${ }^{\circledR}$ inhalation aid is suitable in providing: ${ }^{[1]}$

- High lung deposition, low throat deposition

- High dosage consistency

- Disinfectable, ergonomic SmartTouch masks

Reference: 1. Laube BL, Janssens HM, de Jongh FHC, et al. What the pulmonary specialist should know about the new inhalation therapies. Eur Respir J 2011;37:1308-1331.
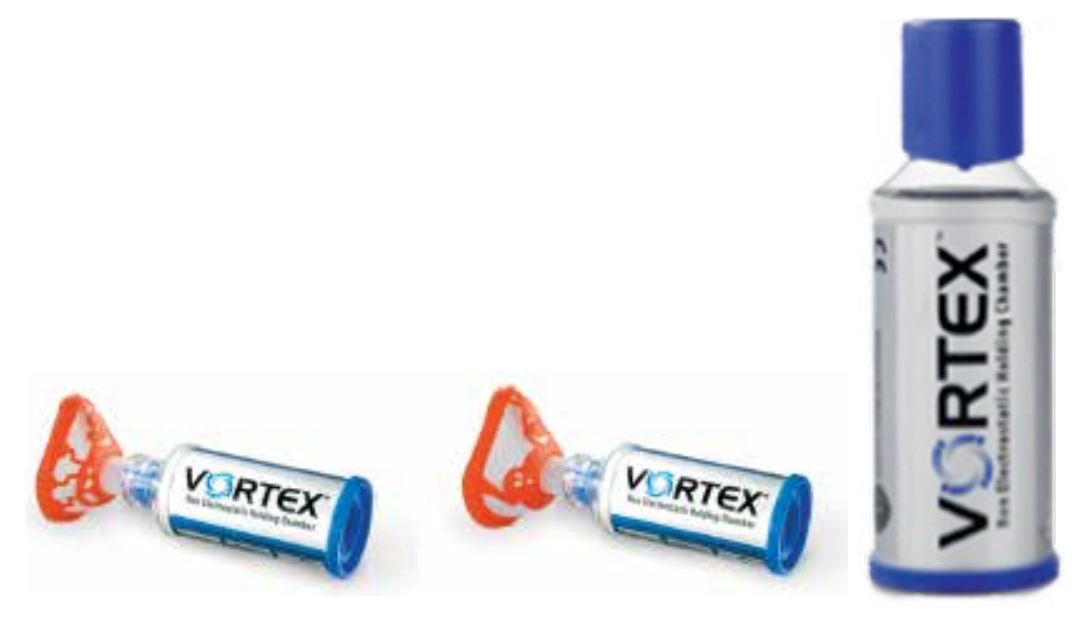
Joint Congress of the South African Thoracic Society $\&$ the South African Society of Sleep Medicine

\section{8 - 21 AUGUST 2016}

The Indaba Hotel \& Conference Centre Johannesburg • South Africa
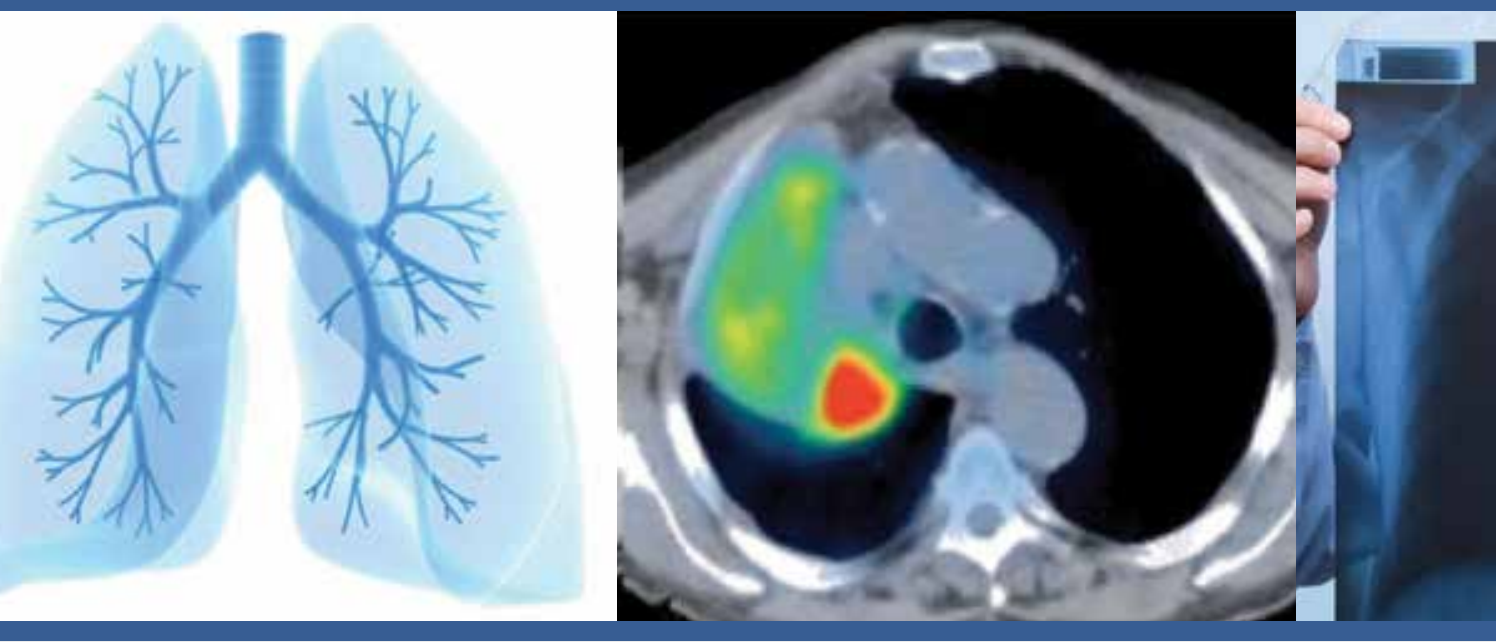

\section{Programme Highlights}

- Pre-congress Workshops

- Sleep Medicine

- The Wheezing Child

- Sleep Apnea \& Cardiometabolic Disease

- Thoracic Surgery Principles

- Critical Care

- Paediatric Pulmonology
- Infectious Diseases \& Neonatal Lung Disease

- Thoracic Surgery Skills 101 \& Thoracic Emergencies

- Interstitial Lung Disease

- Airway Diseases

- Physiotherapy

- Hot Topics

\section{International Faculty}

James Chalmers - United Kingdom

Diego Gonzales Rivas - Spain Karin Klooster - Netherlands Walter McNicholas - Ireland Alan Sihoe - Hong Kong
Eric Simões - USA

Dirk-Jan Slebos - Netherlands

Thierry Troosters - Belgium

Athol Wells- United Kingdom

To view the full scientific programme and register online visit www.satscongress2016.co.za or www.sassmcongress2016.co.za

\section{Congress Office:}

Europa Organisation Africa

Tel +27(0)113250020

enquiries@eoafrica.co.za

www.eoafrica.co.za
SOUTH AFRICAN

SASSM SOCIETY OF SLEEP MEDICINE 


\section{NEW small, solid and effective}

Aspen is proud to announce the launch of an innovative NEW aluminium antistatic holding chamber, as an addition to our respiratory portfolio.

VORTEX ${ }^{\circledR}$ inhalation aid is suitable in providing: (1)

- High lung deposition, low throat deposition

- High dosage consistency

- Disinfectable, ergonomic SmartTouch masks

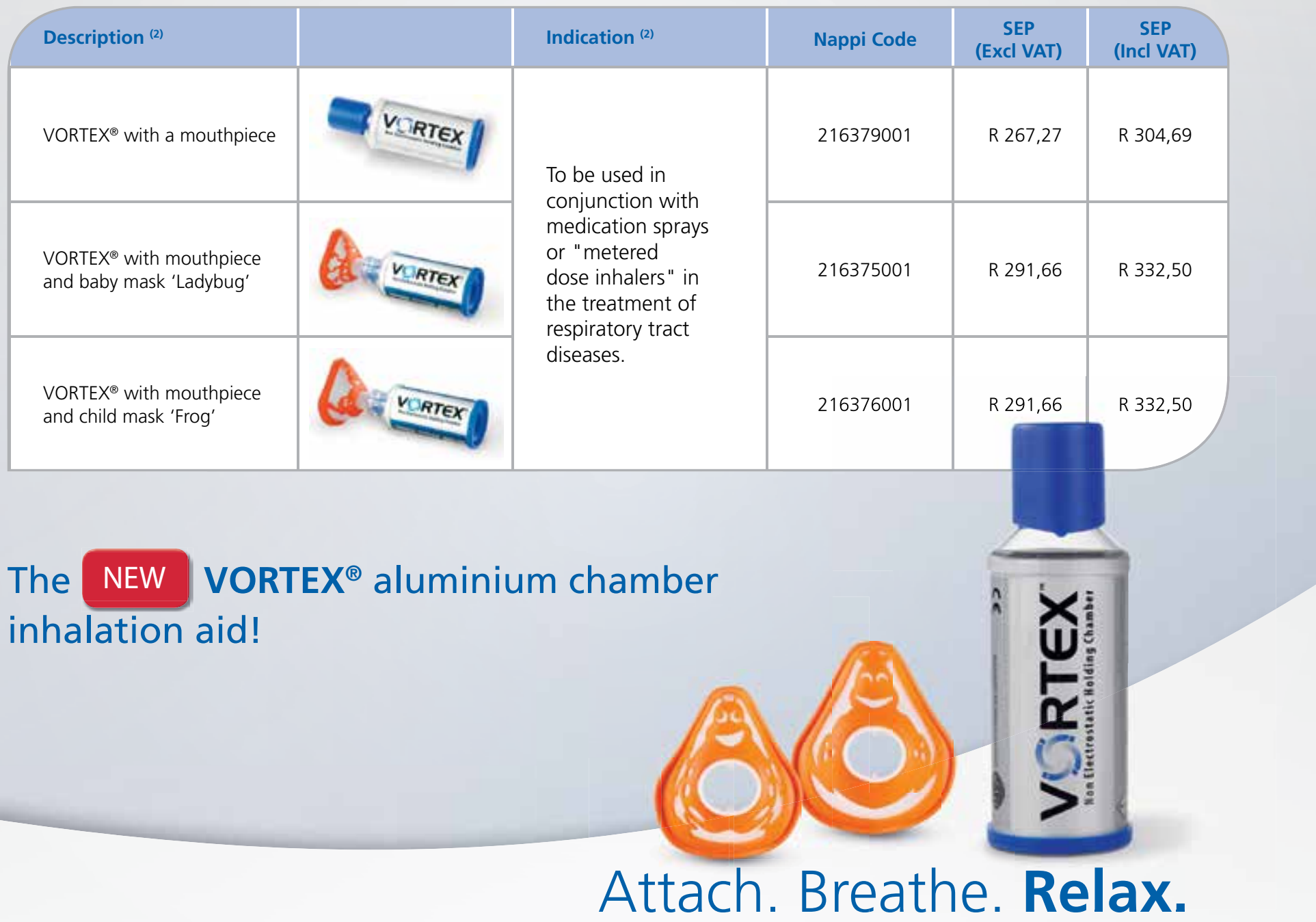

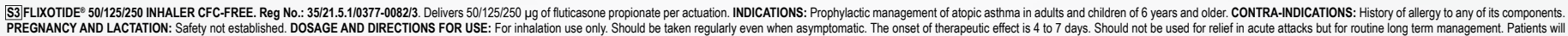
require a fast- and short-acting inhaled bronchodilator to relieve acute symptoms. If patients find that relief with short-acting bronchodilator treatment becomes less effective or they need more inhalations than usual, medical attention must be sought. Adults and children over 16 years of age: $100-1000 \mathrm{\mu g}$ twice daily. Starting dose should be appropriate for severity of the disease. Dose may ber

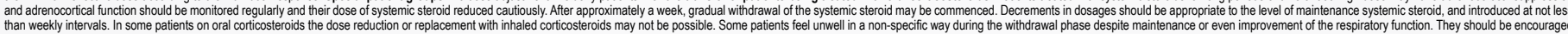
to persevere with inhaled fluticasone propionate and to continue withdrawal of systemic steroid, unless there are objective signs of adrenal insufficieiency. SIIDE EFFECTS AND SPECIAL PRECAUTIONS: Treatment should not be stopped abruptity as adrenal insuffficiency may be precipititeded. Candidiasis of the mouth an Treatment should be discontinued immediately, the patient assessed, and if necessary alternative therapy instituted. Cutaneous hypersensitivity. Systemic corticosteroid effects may occur. Patients transferred from other inhaled steroids or oral steroids remain at risk of impaired adrenal reserve for a considerable time after transferring to inhaled fluticasone propionate. Increasing use to control symptoms indicates deterioration of asthma control and patient should be reassessed. Sudden and progressive deterioration in asthma control is potentially life-threatening and may have several causes. Consideration should be given to increasing corticosteroid dosage if not caused by otherwise treatable causes of deterioration. Severe asthma requires regular medical assessment as death may occur. Sudden worsening of symptoms may require increased corticosteroid dosage which should be administered under urgent medical supervision. Patients weaned eosinophililic condititions (e.g. Churg Strauss syndrome). These cases have usually been associated with reduction or withdrawal of oral corticosteroid therapy. Similarly replacement of systemic steroid treatment with inhaled therapy may unmask allergies such as allergic chinitis or eczzema previously controlled by the systemic
drug. These allergies should be symptomatically treated with antihistamine and/or topical preparations, including topical steroids. Patients in a medical or surgical emergency, who require high doses of inhaled steroids and/or intermittent treatment with oral steroids, are at risk of impaired adrenal reserve. The extent of the adrenal impairment may require specialist advice before elective procedures. The possibility of residual impaired adrenal response and elective situations likely to produce stress and appropriate corticosterolid treatment must be considered. Lack of response or severere exacerbations of asthma should be treated by increasing

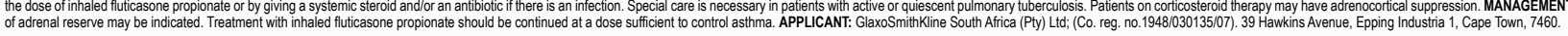
Reference: 1. Laube BL, Janssens HM, de Jongh FHC, et al. What the pulmonary specialist should know about the new inhalation therapies. Eur Respir J 2011:37:1308-1331. 2. VORTEX ${ }^{\circledR}$ package insert. For full prescribing information, please refer to the package inserts approved by the Medicines Regulatory Authority All adverse events should be reported by calling the Aspen Medical Hottine number or directly to GlaxoSmithKline on +27 117456000

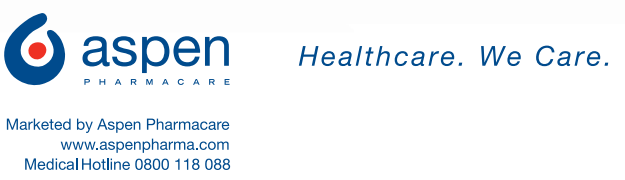

